PROCEEDINGS OF THE

AMERICAN MATHEMATICAL SOCIETY

Volume 127, Number 6, Pages 1651-1663

S 0002-9939(99)04859-5

Article electronically published on February 16, 1999

\title{
AUTOMATIC EXTENSIONS OF LOCAL REGULARIZED SEMIGROUPS AND LOCAL REGULARIZED COSINE FUNCTIONS
}

\author{
SHENG WANG WANG AND MING CHU GAO \\ (Communicated by David R. Larson)
}

\begin{abstract}
This paper establishes automatic extensions for local regularized semigroups and local regularized cosine functions in a certain sense and applies the results to abstract Cauchy problems.
\end{abstract}

\section{INTRODUCTION}

Let $X$ be a complex Banach space and let $A$ be a closed linear operator on $X$. Many physical problems may be modelled as a first or second order abstract Cauchy problem:

$$
u^{\prime}(t)=A u(t)(0 \leq t \leq \tau) ; \quad u(0)=x \quad(A C P 1, \tau)
$$

or

$$
u^{\prime \prime}(t)=A u(t)(-\tau \leq t \leq \tau) ; u(0)=x \quad(A C P 2, \tau) .
$$

The first or second order abstract Cauchy problem is well-posed if $A$ generates a strongly continuous semigroup or cosine function. Then $(A C P 1, \tau)$ or $(A C P 2, \tau)$ has a unique mild solution for all initial data $x$ that extends to a global solution, or equivalently, $\tau$ may be chosen to be $\infty$.

For ill-posed abstract Cauchy problems, the distinction between local and global solutions becomes quite significant. For any given $\tau>0$, it is not hard to construct an operator $A$ for which $(A C P 1, \tau)$ or $(A C P 2, \tau)$ has a unique mild solution for all $x \in D(A)$ but $(A C P 1, n \tau)$ or $(A C P 2, n \tau)$ has no nontrivial solution for every $n>1$ (see examples in [T-O], [H-Hu], [S] and Section 4 of this paper).

In this paper, for $C$ a bounded injective operator on $X$, we show that if $(A C P 1, \tau)$ (resp. $(A C P 2, \tau))$ has a unique mild solution for $x \in \operatorname{Im}(C)$, then for any $n \in N$, $(A C P 1, n \tau)$ (resp. $(A C P 2, n \tau))$ has a unique mild solution for all $x \in \operatorname{Im}\left(C^{n}\right)$ (resp. for all $x, y \in \operatorname{Im}\left(C^{n}\right)$ ). By choosing $C \equiv(\lambda-A)^{-k}$ for $\lambda \in \rho(A)$, this generalizes the result in $[\mathrm{A}-\mathrm{El}-\mathrm{K}]$.

To reach our target, we first study the automatic extensions of local regularized semigroups and local regularized cosine functions in a sense similar to that appearing in $[\mathrm{A}-\mathrm{El}-\mathrm{K}]$, then we consider their applications to both $(A C P 1, n \tau)$ and $(A C P 2, n \tau)$. We also characterize those local regularized semigroups and local regularized cosine functions that can be extended to global ones (see $[\mathrm{G}]$ ). The

Received by the editors May 1, 1997.

1991 Mathematics Subject Classification. Primary 47D05, 47F05.

(C)1999 American Mathematical Society 
approach applied in this paper seems to be more natural, direct and general than the approach applied in [A-El-K].

As regards the theory of local regularized semigroups and local regularized cosine functions, we refer the reader to the references [T-O], $[\mathrm{S}]$ and $[\mathrm{H}-\mathrm{Hu}]$.

The first author of this paper would like to gratefully acknowledge Ralph deLaubenfels for many helpful discussions.

In addition to the complex Banach space $X$, we use $B(X)$ to denote the algebra of all bounded linear operators on $X . C \in B(X)$ is injective and $A$ is closed on $X$. For a linear operator $S, D(S), \operatorname{Im}(S)$ are the domain and image of $S$, respectively. $\rho(S), \sigma(S)$ are the resolvent set and spectrum of $S$, respectively.

Given $\tau>0, n \in N$ and $x \in X$. Consider the first order abstract Cauchy problem $(A C P 1, n \tau)$. By a mild solution of $(A C P 1, n \tau)$ we mean a map $t \mapsto$

$u_{n}(t) \in C([0, n \tau], X)$ such that $v_{n}(t) \equiv \int_{0}^{t} u_{n}(s) d s \in D(A)$ for all $t \in[0, n \tau]$ and satisfies

$$
\frac{d}{d t} v_{n}(t)=A\left[v_{n}(t)\right]+x \quad(t \in[0, n \tau]) .
$$

$(A C P 1, n \tau)$ is said to be $C^{n}$-well-posed, if it has a unique mild solution $u_{n} \in$ $C([0, n \tau], X)$ for every $x \in \operatorname{Im}\left(C^{n}\right)$.

In addition to $(A C P 1, n \tau)$, we shall also consider the second order abstract Cauchy problem $(A C P 2, n \tau)$. Let $x, y \in X$. By a mild solution of $(A C P 2, n \tau)$ we mean a map $t \mapsto u_{n}(t) \in C([-n \tau, n \tau], X)$ such that $w_{n}(t) \equiv \int_{0}^{t}(t-s) u_{n}(s) d s \in$ $D(A)$ for all $t \in[-n \tau, n \tau]$ and satisfies

$$
\left(\frac{d}{d t}\right)^{2} w_{n}(t)=A\left(w_{n}(t)\right)+x+t y \quad(t \in[-n \tau, n \tau]) .
$$

$(A C P 2, n \tau)$ is said to be $C^{n}$-well-posed, if it has a unique mild solution $u_{n} \in$ $C([-n \tau, n \tau], X)$ for every pair of $x, y \in \operatorname{Im}\left(C^{n}\right)$.

In Section 2, we study the automatic extension of local regularized semigroups and their applications to $(A C P 1, n \tau)$. In Section 3 , we consider the automatic extension of local regularized cosine functions and their applications to $(A C P 2, n \tau)$. It is surprising that all local regularized semigroups and all local regularized cosine functions can be extended in our weaker sense without any additional conditions. In Section 4, we offer several examples to illustrate the main results obtained in Sections 2 and 3.

\section{Extension of LOCAL $C$-REgUlarized SEMigroups}

The following definition can be found in [T-O].

Definition 2.1. Assume $C \in B(X)$ is injective and $\tau>0$. A strongly continuous family $\{W(t)\}_{t \in[0, \tau]}$ of bounded operators is a local $C$-regularized semigroup if

(i) $W(0)=C$;

(ii) $W(s) W(t)=W(s+t) C$ for all $0 \leq s, t, s+t \leq \tau$.

$\{W(t)\}_{t \in[0, \tau]}$ is nondegenerate if $W(t) x \equiv 0$, for all $t \in[0, \tau]$, only when $x=0$. If $\{W(t)\}_{t \in[0, \tau]}$ is nondegenerate, its generator $A$ is defined to be the following operator:

$$
x \in D(A) \text { and } y=A x \Longleftrightarrow W(t) x=\int_{0}^{t} W(s) y d s+C x, \forall t \in[0, \tau] .
$$


An equivalent definition for generators of regularized semigroups can be found in $[\mathrm{dL} 1]$.

The following lemma is an analogue of [dL 3-S-Wa, Proposition 2.2].

Lemma 2.2. The local $C$-regularized semigroup $\{W(t)\}_{t \in[0, \tau]}$ is nondegenerate if and only if $C$ is injective.

Definition 2.3. Assume $A$ is closed, $C \in B(X)$ is injective and $\tau>0$. A strongly continuous family $\{W(t)\}_{t \in[0, \tau]}$ of bounded operators is a local mild $C$-existence family for $A$ if

(i) $\int_{0}^{t} W(s) x d s \in D(A)$ and $t \mapsto A \int_{0}^{t} W(s) x d s$ is continuous on $[0, \tau]$ for all $x \in X$;

(ii) $A \int_{0}^{t} W(s) x d s=W(t) x-C x$ for all $x \in X, t \in[0, \tau]$.

The concept of a global mild $C$-existence family for $A$ was first introduced in [dL 2] and summarized in [dL 1].

A local $C$-regularized semigroup $\{W(t)\}_{t \in[0, \tau]}$, satisfying (i), (ii) of Definition 2.3 and $W(t) A \subseteq A W(t)$ for $t \in[0, \tau]$, is said to be a local $C$-regularized semigroup for $A$.

It has been proved in [dL 3-S-Wa, Proposition 2.9, Proposition 2.6 (2)] that a global $C$-regularized semigroup for $A$ is uniquely determined by $A$ and that if a global $C$-regularized semigroup is generated by $A$, then it is for $A$. It is routine to verify that these facts remain true for the local case. Hence, instead of the term: $a$ local $C$-regularized semigroup for $A$, we will say the local $C$-regularized semigroup for $A$.

The following lemma for local $C$-regularized semigroups is similar to the global case (see [dL 3-S-Wa, Theorem 3.3]) and plays an important role in this section.

Lemma 2.4. Assume $A$ is closed, $C \in B(X)$ is injective and $C A \subseteq A C$. Then the following are equivalent.

(a) $(A C P 1, \tau)$ is $C$-well-posed.

(b) There exists a local mild $C$-existence family $\{W(t)\}_{t \in[0, \tau]}$ for $A$ so that $W(t) A$ $\subseteq A W(t)$ for $t \in[0, \tau]$.

(c) There exists a local $C$-regularized semigroup $\{W(t)\}_{t \in[0, \tau]}$ for $A$.

Assume $\{W(t)\}_{t \in[0, \tau]}$ is a local $C$-regularized semigroup. Denote

$$
W_{1}(t) \equiv W(t), \forall t \in[0, \tau] .
$$

We define the family $\left\{W_{n}(t)\right\}_{t \in[0, n \tau]}$ of bounded operators by induction in $n \in N$. Assume the family $\left\{W_{n-1}(t)\right\}_{t \in[0,(n-1) \tau]}$ has already been defined. Now define

$$
W_{n}(t) \equiv \begin{cases}W_{n-1}(t) C, & \forall t \in[0,(n-1) \tau] \\ W_{1}(t-(n-1) \tau) W_{n-1}((n-1) \tau), & \forall t \in[(n-1) \tau, n \tau]\end{cases}
$$

The following corollary is an immediate consequence of the definition of $W_{n}(\cdot)$.

Corollary 2.5. For every $n \in N,\left\{W_{n}(t)\right\}_{t \in[0, n \tau]}$ is strongly continuous, $W_{n}(t) A$ $\subseteq A W_{n}(t)$, and $W_{n}(t)=C^{k} W_{n-k}(t)$ for $t \in[0,(n-k) \tau], k=0,1, \ldots, n-1$.

Theorem 2.6. Assume $A$ is closed, $C \in B(X)$ is injective and $\{W(t)\}_{t \in[0, \tau]}$ is a local $C$-regularized semigroup. Then the following hold.

(a) $\left\{W_{n}(t)\right\}_{t \in[0, n \tau]}$ is a local $C^{n}$-regularized semigroup for every $n \in N$.

(b) If $\{W(t)\}_{t \in[0, \tau]}$ is for (resp. generated by) A, then $\left\{W_{n}(t)\right\}_{t \in[0, n \tau]}$ is also for every $n \in N$. 
Proof. As above, denote $W_{1}(t) \equiv W(t)$ for $t \in[0, \tau]$. Assume $\left\{W_{1}(t)\right\}_{t \in[0, \tau]}$ is for $A$ and (a) is true for some $n-1$ with $n>1$. First assume $t \in[0,(n-1) \tau]$. Then

$$
\begin{aligned}
& A \int_{0}^{t} W_{n}(s) x d s=A \int_{0}^{t} W_{n-1}(s) C x d s \\
& \quad=W_{n-1}(t) C x-C^{n} x=W_{n}(t) x-C^{n} x, \forall x \in X .
\end{aligned}
$$

Now assume $t \in[(n-1) \tau, n \tau]$. Then

$$
\begin{aligned}
A & \int_{0}^{t} W_{n}(s) x d s=A \int_{0}^{(n-1) \tau} W_{n}(s) x d s+A \int_{(n-1) \tau}^{t} W_{n}(s) x d s \\
& =W_{n-1}((n-1) \tau) C x-C^{n} x+A \int_{0}^{t-(n-1) \tau} W_{n}(s+(n-1) \tau) x d s \\
& =W_{n-1}((n-1) \tau) C x-C^{n} x+W_{n-1}((n-1) \tau) A \int_{0}^{t-(n-1) \tau} W_{1}(s) x d s \\
& =W_{n-1}((n-1) \tau) C x-C^{n} x+W_{n-1}((n-1) \tau)\left[W_{1}(t-(n-1) \tau) x-C x\right] \\
& =W_{n}(t) x-C^{n} x .
\end{aligned}
$$

From Corollary 2.5 and Lemma 2.4, $\left\{W_{n}(t)\right\}_{t \in[0, n \tau]}$ is a local $C^{n}$-regularized semigroup for $A$.

Now assume $A$ is the generator of $\{W(t)\}_{t \in[0, \tau]}$. Then $A=C^{-1} A C$ by $[\mathrm{dL} 1$, Proposition 3.11] or [dL 3-S-Wa, Propositiion 2.9]. Here we note that these references are for global regularized semigroups, but the same proof applies to the local case. By induction in $n$, it is easy to show that $A=C^{-n} A C^{n}$ for every $n \in N$. This, together with (2.1) and (2.2), asserts that $A$ is the generator of $\left\{W_{n}(t)\right\}_{t \in[0, n \tau]}$ by the above references again.

It is easy to see that if $\|W(t)\| \leq M$ for $t \in[0, \tau]$ and some $M>0$, then $\left\|W_{n}(t)\right\| \leq M^{n}$ for all $t \in[0, n \tau]$ and $n \in N$.

The following corollary is an easy consequence of Theorem 2.6 and Lemma 2.4.

Corollary 2.7. Assume $A$ is closed and $C A \subseteq A C$. Then the following are equivalent.

(a) $(A C P 1, \tau)$ is $C$-well-posed.

(b) There exists a local $C$-regularized semigroup $\{W(t)\}_{t \in[0, \tau]}$ for $A$.

(c) $(A C P 1, n \tau)$ is $C^{n}$-well-posed for every $n \in N$.

(d) There exists a local $C^{n}$-regularized semigroup $\left\{W_{n}(t)\right\}_{t \in[0, n \tau]}$ for A for every $n \in N$.

Corollary 2.8. Assume $A$ is closed, $C \in B(X)$ is injective and $\{W(t)\}_{t \in[0, \tau]}$ is a local $C$-regularized semigroup. Then the following are equivalent.

(a) $\{W(t)\}_{t \in[0, \tau]}$ can be extended to a global $C$-regularized semigroup.

(b) For every $n \in N$,

$$
\operatorname{Im}\left(W(t)[W(\tau)]^{n-1}\right) \subseteq \operatorname{Im}\left(C^{n-1}\right)
$$

and $C^{-(n-1)} W(t)[W(\tau)]^{n-1}$ is strongly continuous for $t \in[0, \tau]$.

(c) For every $n \in N$,

$$
\operatorname{Im}\left(W_{n}(t)\right) \subseteq \operatorname{Im}\left(C^{n-1}\right)
$$

and $C^{-(n-1)} W_{n}(t)$ is strongly continuous for $t \in[0, n \tau]$. 
When one of the equivalent conditions (a), (b) or (c) is true, denote the global extension of $\{W(t)\}_{t \in[0, \tau]}$ by $\left\{W_{0}(t)\right\}_{t \in[0, \infty)}$. If $\{W(t)\}_{t \in[0, \tau]}$ is for (resp. generated by) A, then $\left\{W_{0}(t)\right\}_{t \in[0, \infty)}$ is also.

Proof. (a) $\Longrightarrow$ (b). Clear.

(b) $\Longrightarrow(\mathrm{c})$. The following hold for $n=1$.

(1) $W_{n}(t)=W_{1}(t-(n-1) \tau)\left[W_{1}(\tau)\right]^{n-1}$ for $t \in[(n-1) \tau, n \tau]$;

(2) $C^{-(n-1)} W_{n}(t)$ is strongly continuous for $t \in[0, n \tau]$.

Now assume (1) and (2) are true for some $n \in N$. Set $t=n \tau$ in (1) to find $W_{n}(n \tau)=\left[W_{1}(\tau)\right]^{n}$. From the definition, we have

$$
W_{n+1}(t)= \begin{cases}W_{n}(t) C, & \forall t \in[0, n \tau] ; \\ W_{1}(t-n \tau)\left[W_{1}(\tau)\right]^{n}, & \forall t \in[n \tau,(n+1) \tau] .\end{cases}
$$

Thus, (2), together with the hypotheses in (b), implies that $\operatorname{Im}\left(W_{n+1}(t)\right) \subseteq \operatorname{Im}\left(C^{n}\right)$ and $C^{-n} W_{n+1}(t)$ is strongly continuous for $t \in[0,(n+1) \tau]$. Hence, $(2)$ is true for $n$ replaced by $n+1$. (c) is proved.

$(\mathrm{c}) \Longrightarrow(\mathrm{a})$. Assume $\left\{W_{1}(t)\right\}_{t \in[0, \tau]}$ is for $A$. Define

$$
W_{0}(t) \equiv C^{-(n-1)} W_{n}(t), \forall t \in[0, n \tau], n \in N .
$$

Then $W_{0}(\cdot)$ is well defined and strongly continuous on $[0, n \tau]$ for every $n \in N$, hence on $[0, \infty)$. (2.1) and $(2.2)$ give us

$$
A \int_{0}^{t} W_{0}(s) x d s=W_{0}(t) x-C x, \forall t \in[0, \infty) .
$$

From Lemma 2.4 and the inclusion $W_{0}(t) A \subseteq A W_{0}(t),\left\{W_{0}(t)\right\}_{t \in[0, \infty)}$ is a $C$ regularized semigroup for $A$. If $\left\{W_{1}(t)\right\}_{t \in[0, \tau]}$ is generated by $A$, then $\left\{W_{0}(t)\right\}_{t \in[0, \infty)}$ is also by its definition and Theorem 2.6 .

The equivalence of (a) and (b) in Corollary 2.8 was proved in [G, Theorem 1.1] by a more complicated method without assuming the strong continuity of $C^{-(n-1)} W(t)$ $[W(\tau)]^{n-1}$ on $[0, \tau]$, which seems to be needed.

Corollary 2.9. Assume $A$ is closed, $\rho(A)$ is nonempty, $r \in \rho(A)$ and $k \in N$. Then the following are equivalent.

(a) $(A C P 1, \tau)$ is $(A-r)^{-k}$-well-posed.

(b) There exists a local $(A-r)^{-k}$-regularized semigroup generated by $A$.

(c) $(A C P 1, n \tau)$ is $(A-r)^{-n k}$-well-posed for every $n \in N$.

(d) There exists a local $(A-r)^{-n k}$-regularized semigroup generated by A for every $n \in N$.

Proof. Set $C \equiv(A-r)^{-k}$ in Corollary 2.7 .

It is worthwhile to mention that [A-El-K, Theorem 4.1] is a consequence of Corollary 2.9. This corollary is also guaranteeing mild solutions of $(A C P 1, n \tau)$ for all $x \in D\left(A^{n k}\right)$ and all $n \in N$, when there are mild solutions of $(A C P 1, \tau)$ for all $x \in D\left(A^{k}\right)$.

The following proposition seems to be interesting.

Proposition 2.10. Assume $0 \in \rho(A)$ and $A$ satisfies the equivalent conditions in Corollary 2.9 with $r=0$. Let $\left\{W_{1}(t)\right\}_{t \in[0, \tau]}$ be the $A^{-k}$-regularized semigroup 
generated by $A$. Then $W_{n+m}(\cdot) x$ is mk-th continuously differentiable on $[0, n \tau]$ for every $x \in X$ and

$$
\left(\frac{d}{d t}\right)^{m k} W_{n+m}(t) x=W_{n}(t) x, \forall t \in[0, n \tau] .
$$

Proof. Assume $t \in[0, n \tau]$ and $x \in X$. From

$$
\begin{aligned}
& A \int_{0}^{t} W_{n}(s) A^{-m k} x d s=W_{n}(t) A^{-m k} x-A^{-(n+m) k} x ; \\
& A \int_{0}^{t} W_{n+m}(s) x d s=W_{n+m}(t) x-A^{-(n+m) k} x
\end{aligned}
$$

and the uniqueness of the solutions of $(A C P 1, n \tau)$, we have

$$
W_{n+m}(t) x=W_{n}(t) A^{-m k} x .
$$

$W_{n+m}(t) x$ is thus $m k$-th continuously differentiable for $t \in[0, n \tau]$ and (2.3) follows.

The following interesting remark was suggested by the referee.

Remark 2.11. In addition to the extension of local $C$-regularized semigroups defined on closed interval $[0, \tau]$, it is also necessary to consider the extension of local $C$ regularized semigroups defined on the interval $[0, \tau)$ (see Example 4.2). Assume that $\left\{W_{1}(t)\right\}_{t \in[0, \tau)}$ is such a local $C$-regularized semigroup and that its extension $\left\{W_{n}(t)\right\}_{t \in[0, n \tau)}$ has been defined and is a local $C^{n}$-regularized semigroup. Now define

$$
W_{n+1}(t) \equiv\left\{\begin{array}{l}
W_{n}(t) C, \quad \forall t \in[0, n \tau) \\
W_{1}(t-s) W_{n}(s), \quad \forall t \in[n \tau,(n+1) \tau],
\end{array}\right.
$$

where $s$ is chosen so that $s \in[(n-1) \tau, n \tau]$ and $t-s<\tau$. We have to show that $W_{1}(t-s) W_{n}(t-s)$ is independent of $s \in[(n-1) \tau, n \tau)$. Let $s^{\prime} \in[(n-1) \tau, n \tau)$ be such that $t-s^{\prime}<\tau$ and $s^{\prime}<s$. Since $s-s^{\prime}<\tau$, we have $W_{n}\left(s-s^{\prime}\right)=W_{1}\left(s-s^{\prime}\right) C^{n-1}$ and hence

$$
\begin{aligned}
W_{n}(s) C^{n} & =W_{n}\left(s^{\prime}+s-s^{\prime}\right) C^{n}=W_{n}\left(s-s^{\prime}\right) W_{n}\left(s^{\prime}\right) \\
& =W_{1}\left(s-s^{\prime}\right) C^{n-1} W_{n}\left(s^{\prime}\right) .
\end{aligned}
$$

This implies

$$
\begin{aligned}
W_{1}(t-s) W_{n}(s) C^{n} & =W_{1}(t-s) W_{1}\left(s-s^{\prime}\right) W_{n}\left(s^{\prime}\right) C^{(n-1)} \\
& =W_{1}\left(t-s^{\prime}\right) W_{n}\left(s^{\prime}\right) C^{n}
\end{aligned}
$$

Thus, $W_{1}(t-s) W_{n}(s)=W_{1}\left(t-s^{\prime}\right) W_{n}\left(s^{\prime}\right)$ by the injectivity of $C$.

It is routine to verify that $\left\{W_{n+1}(t)\right\}_{t \in[0,(n+1) \tau)}$ is a local $C^{n+1}$ - regularized semigroup, and if $\left\{W_{1}(t)\right\}_{t \in[0, \tau)}$ is for (resp. generated by) $A$, then $\left\{W_{n+1}(t)\right\}_{t \in[0,(n+1) \tau)}$ is also for every $n \in N$.

\section{Extension of LOCAL $C$-REgUlarized Cosine FunCtions}

The extension of local $C$-regularized cosine functions is similar to that of local $C$-regularized semigroups except for some minor differences. We give the details for completeness. 
Definition 3.1. Assume $C \in B(X)$ is injective and $\tau>0$. A strongly continuous family $\{\mathbb{C}(t)\}_{t \in[-\tau, \tau]}$ is a local $C$-regularized cosine function if

(i) $\mathbb{C}(0)=C$;

(ii) $\mathbb{C}(s+t) C+\mathbb{C}(s-t) C=2 \mathbb{C}(s) \mathbb{C}(t), \forall s, t, s \pm t \in[-\tau, \tau]$.

$\{\mathbb{C}(t)\}_{t \in[-\tau, \tau]}$ is nondegenerate if $\mathbb{C}(t) x \equiv 0$, for all $t \in[-\tau, \tau]$, only when $x=0$. If $\{\mathbb{C}(t)\}_{t \in[-\tau, \tau]}$ is nondegenerate, the generator $A$ of $\{\mathbb{C}(t)\}_{t \in[\tau, \tau]}$ is defined to be the following operator:

$$
x \in D(A) \text { and } y=A x \Longleftrightarrow \mathbb{C}(t) x=\int_{0}^{t}(t-s) \mathbb{C}(s) y d s+C x, \forall t \in[-\tau, \tau] .
$$

The following lemma is a version of [W-Wa, Propoition 2.2].

Lemma 3.2. The local C-regularized cosine function $\{\mathbb{C}(t)\}_{t \in[-\tau, \tau]}$ is nondegenerate if and only if $C$ is injective.

Similar to Definition 2.3, we introduce the following.

Definition 3.3. Assume $A$ is closed, $C \in B(X)$ is injective and $\tau>0$. A strongly continuous family $\{\mathbb{C}(t)\}_{t \in[-\tau, \tau]}$ of bounded operators is a local second-order mild $C$-existence family for $A$ if

(i) $\int_{0}^{t}(t-s) \mathbb{C}(s) x d s \in D(A)$ and $t \mapsto A \int_{0}^{t}(t-s) \mathbb{C}(s) x d s$ is continuous on $[-\tau, \tau]$ for all $x \in X$.

(ii) $A \int_{0}^{t}(t-s) \mathbb{C}(s) x d s=\mathbb{C}(t)-C x$, for all $t \in[-\tau, \tau]$.

A local $C$-regularized cosine function $\{\mathbb{C}(t)\}_{t \in[-\tau, \tau]}$, satisfying (i), (ii) of Definition 3.3 and $\mathbb{C}(t) A \subseteq A \mathbb{C}(t)$ for $t \in[-\tau, \tau]$, is called a local $C$-regularized cosine function for $A$. Since [W-Wa, Theorem 2.6 (1), Corollary 2.8] remain true for our local case, if $\{\mathbb{C}(t)\}_{t \in[-\tau, \tau]}$ is generated by $A$, then it is for $A$ and, instead of the term: a local $C$-regularized cosine function for $A$, we may say the local $C$-regularized cosine function for $A$.

The following lemma for local $C$-regularized cosine functions is similar to the global case (see [W-Wa, Theorem 3.4]) and plays an important role in this section.

Lemma 3.4. Assume $A$ is closed, $C \in B(X)$ is injective and $C A \subseteq A C$. Then the following are equivalent.

(a) $(A C P 2, \tau)$ is $C$-well-posed.

(b) There exists a local second-order mild $C$-existence family $\{\mathbb{C}(t)\}_{t \in[-\tau, \tau]}$ for $A$ so that $\mathbb{C}(t) A \subseteq A \mathbb{C}(t)$ for $t \in[-\tau, \tau]$.

(c) There exists a local $C$-regularized cosine function $\{\mathbb{C}(t)\}_{t \in[-\tau, \tau]}$ for $A$.

Let $\{\mathbb{C}(t)\}_{t \in[-\tau, \tau]}$ be a local $C$-regularized cosine function. Denote $\mathbb{C}_{1}(t) \equiv$ $\mathbb{C}(t)$. As with section 2, assume the family $\left\{\mathbb{C}_{n-1}(t)\right\}_{t \in[-(n-1) \tau,(n-1) \tau]}$ of bounded operators has been defined for some $n>1$. Now define

$$
\mathbb{C}_{n}(t) \equiv \begin{cases}\mathbb{C}_{n-1}(t) C, & \forall t \in[0,(n-1) \tau] ; \\ 2 \mathbb{C}_{1}(t-(n-1) \tau) \mathbb{C}_{n-1}((n-1) \tau) & \\ -\mathbb{C}_{n-1}(2(n-1) \tau-t) C, & \forall t \in[(n-1) \tau, n \tau] ; \\ \mathbb{C}_{n}(-t), & \forall t \in[-n \tau, 0] .\end{cases}
$$

The following corollary is also an immediate consequence of the definition of $\mathbb{C}_{n}(\cdot)$.

Corollary 3.5. For each $n \in N,\left\{\mathbb{C}_{n}(t)\right\}_{t \in[-n \tau, n \tau]}$ is strongly continuous, $\mathbb{C}_{n}(t) A$ $\subseteq A \mathbb{C}_{n}(t)$ and $\mathbb{C}_{n}(t)=C^{k} \mathbb{C}_{n-k}(t), \forall t \in[-(n-k) \tau,(n-k) \tau], k=0,1, \ldots, n-1$ 
Theorem 3.6. Assume $A$ is closed, $C$ is injective and $\{\mathbb{C}(t)\}_{t \in[-\tau, \tau]}$ is a local $C$-regularized cosine function. Then the following hold.

(a) $\left\{\mathbb{C}_{n}(t)\right\}_{t \in[-n \tau, n \tau]}$ is a local $C^{n}$-regularized cosine function for every $n \in N$.

(b) If $\{\mathbb{C}(t)\}_{t \in[-\tau, \tau]}$ is for (resp. generated by) A, then $\left\{\mathbb{C}_{n}(t)\right\}_{t \in[-n \tau, n \tau]}$ is also for every $n \in N$.

Proof. Denote $\mathbb{C}_{1}(t) \equiv \mathbb{C}(t)$ for $t \in[-\tau, \tau]$. Assume $\left\{\mathbb{C}_{1}(t)\right\}_{t \in[-\tau, \tau]}$ is for $A$ and (a) is true for some $n-1$ with $n>1$. First assume $t \in[0,(n-1) \tau]$. Then

$$
\begin{aligned}
& A \int_{0}^{t}(t-s) \mathbb{C}_{n}(s) x d s=A \int_{0}^{t}(t-s) \mathbb{C}_{n-1}(s) C x d s \\
& =\mathbb{C}_{n-1}(t) C x-C^{n} x=\mathbb{C}_{n}(t) x-C^{n} x, \forall x \in X .
\end{aligned}
$$

Now assume $t \in[(n-1) \tau, n \tau]$. Then

$$
\begin{aligned}
\int_{0}^{t}(t-s) \mathbb{C}_{n}(s) C x d s & =\int_{0}^{(n-1) \tau}(t-s) \mathbb{C}_{n-1}(s) C x d s \\
& +2 \mathbb{C}_{n-1}((n-1) \tau) \int_{(n-1) \tau}^{t}(t-s) \mathbb{C}_{1}(s-(n-1) \tau) x d s \\
& -\int_{(n-1) \tau}^{t}(t-s) \mathbb{C}_{n-1}(2(n-1) \tau-s) C x d s .
\end{aligned}
$$

Since

$$
\begin{aligned}
\int_{0}^{(n-1) \tau}( & t-s) \mathbb{C}_{n-1}(s) C x d s \\
& =2 \int_{0}^{(n-1) \tau}((n-1) \tau-s) \mathbb{C}_{n-1}(s) C x d s \\
& -\int_{0}^{(n-1) \tau}\left[((2(n-1) \tau-t)-s] \mathbb{C}_{n-1}(s) C x d s ;\right. \\
\int_{(n-1) \tau}^{t}( & t-s) \mathbb{C}_{n-1}(2(n-1) \tau-s) C x d s \\
& =-\int_{(n-1) \tau}^{2(n-1) \tau-t}[(2(n-1) \tau-t)-s] \mathbb{C}_{n-1}(s) C x d s ; \\
2 \mathbb{C}_{n-1}((n-1) \tau) \int_{(n-1) \tau}^{t}(t-s) \mathbb{C}_{1}(s-(n-1) \tau) x d s & \\
= & 2 \mathbb{C}_{n-1}((n-1) \tau) \int_{0}^{t-(n-1) \tau}[t-(n-1) \tau-s] C_{1}(s) x d s
\end{aligned}
$$

it follows that

$$
\begin{aligned}
& A \int_{0}^{t}(t-s) \mathbb{C}_{n}(s) x d s \\
& \quad=2 \mathbb{C}_{n-1}((n-1) \tau) C x-2 C^{n} x-\mathbb{C}_{n-1}(2(n-1) \tau-t) C x+C^{n} x \\
& \quad+2 \mathbb{C}_{(n-1)}((n-1) \tau)\left[\mathbb{C}_{1}(t-(n-1) \tau) x-C x\right] \\
& \quad=2 \mathbb{C}_{(n-1)}((n-1) \tau) \mathbb{C}_{1}(t-(n-1) \tau) x-\mathbb{C}_{n-1}(2(n-1) \tau-t) C x-C^{n} x \\
& \quad=\mathbb{C}_{n}(t) x-C^{n} x, \quad \forall x \in X .
\end{aligned}
$$


From $C_{n}(t)=\mathbb{C}_{n}(-t)$ for $t \in[-n \tau, 0],(3.1)$ and (3.2) remain true for all $t \in$ $[-n \tau, n \tau]$. Corollary 3.5 and Lemma 3.4 imply that $\left\{\mathbb{C}^{n}(t)\right\}_{t \in[-n \tau, n \tau]}$ is the local $C^{n}$-regularized cosine function for $A$.

By Lemma 3.2, Corollary 3.5 and an argument identical to that of Theorem 2.6, it follows that if $\{\mathbb{C}(t)\}_{t \in[-\tau, \tau]}$ is generated by $A$, then $\left\{\mathbb{C}_{n}(t)\right\}_{t \in[-n \tau, n \tau]}$ is also for every $n \in N$.

Corollary 3.7. Assume $A$ is closed, $C A \subseteq A C$ and $\tau>0$. Then the following are equivalent.

(a) $(A C P 2, \tau)$ is $C$-well-posed.

(b) There exists a local C-regularized cosine function $\{\mathbb{C}(t)\}_{t \in[-\tau, \tau]}$ for $A$.

(c) $(A C P 2, n \tau)$ is $C^{n}$-well-posed for every $n \in N$.

(d) There exists a local $C^{n}$-regularized cosine function $\left\{\mathbb{C}_{n}(t)\right\}_{t \in[-n \tau, n \tau]}$ for A for every $n \in N$.

Corollary 3.8. Assume $A$ is closed, $C \in B(X)$ is injective and $\{\mathbb{C}(t)\}_{t \in[-\tau, \tau]}$ is a local $C$-regularized cosine function. Then the following are equivalent.

(a) $\{\mathbb{C}(t)\}_{t \in[-\tau, \tau]}$ can be extended to a global $C$-regularized cosine function.

(b) For every $n \in N, \operatorname{Im}\left(\mathbb{C}(t)[\mathbb{C}(\tau)]^{n-1}\right) \subseteq \operatorname{Im}\left(C^{n-1}\right)$ and $C^{-(n-1)} \mathbb{C}(t)[\mathbb{C}(\tau)]^{n-1}$ is strongly continuous for $t \in[-\tau, \tau]$.

(c) For every $n \in N, \operatorname{Im}\left(\mathbb{C}_{n}(t)\right) \subseteq \operatorname{Im}\left(C^{n-1}\right)$ and $C^{-(n-1)} \mathbb{C}_{n}(t)$ is strongly continuous for $t \in[-n \tau, n \tau]$.

When one of the equivalent conditions (a), (b) or (c) holds, denote the global extension of $\{\mathbb{C}(t)\}_{t \in[-\tau, \tau]}$ by $\left\{\mathbb{C}_{0}(t)\right\}_{t \in(-\infty, \infty)}$. If $\{\mathbb{C}(t)\}_{t \in[-\tau, \tau]}$ is for (resp. generated by) A, then $\left\{\mathbb{C}_{0}(t)\right\}_{t \in(-\infty, \infty)}$ is also.

Proof. (a) $\Longrightarrow$ (b). Clear.

(b) $\Longrightarrow(\mathrm{c})$. To prove (c), it suffices to prove the following.

(1) Both $\mathbb{C}_{n}((n-1) \tau), \mathbb{C}_{n}(n \tau)$ are homogeneous polynomials of $\mathbb{C}_{1}(\tau)$ and $C$ of degree $n$.

(2) $\operatorname{Im}\left(\mathbb{C}_{n}(t)\right) \subseteq \operatorname{Im}\left(C^{n-1}\right), C^{-(n-1)} \mathbb{C}_{n}(t)$ is strongly continuous for $t \in[0, n \tau]$.

(1) and (2) hold for $n=1$. Now assume they are true for some $n \in N$. By definition,

$$
\begin{aligned}
& \mathbb{C}_{n+1}(n \tau)=\mathbb{C}_{n}(n \tau) C ; \\
& \mathbb{C}_{n+1}((n+1) \tau)=2 \mathbb{C}_{1}(\tau) \mathbb{C}_{n}(n \tau)-\mathbb{C}_{n}((n-1) \tau) C .
\end{aligned}
$$

(1) is thus true for $n$ replaced by $n+1$. From (1) and (b), we have

(3) $\operatorname{Im}\left(\mathbb{C}_{1}(t-n \tau) \mathbb{C}_{n}(n \tau)\right) \subseteq \operatorname{Im}\left(C^{n}\right)$ and $C^{-n} \mathbb{C}_{1}(t-n \tau) \mathbb{C}_{n}(n \tau)$ is strongly continuous for $t \in[n \tau,(n+1) \tau]$.

(2), which holds by our inductive hypothesis, gives us that

(4) $\operatorname{Im}\left(\mathbb{C}_{n}(2 n \tau-t)\right) \subseteq \operatorname{Im}\left(C^{n-1}\right)$ and $C^{-(n-1)} \mathbb{C}_{n}(2 n \tau-t)$ is strongly continuous for $t \in[n \tau,(n+1) \tau]$, since for this case we have $(2 n \tau-t) \in[(n-1) \tau, n \tau]$.

The definition, together with (2), (3) and (4), implies that $\operatorname{Im}\left(\mathbb{C}_{n+1}(t)\right) \subseteq$ $\operatorname{Im}\left(C^{n}\right)$ and $C^{-n} \mathbb{C}_{n+1}(t)$ is strongly continuous for $t \in[0,(n+1) \tau]$. (2) holds for every $n \in N$. (c) is proved. 
(c) $\Longrightarrow$ (a). Assume $\left\{\mathbb{C}_{1}(t)\right\}_{t \in[-\tau, \tau]}$ is for $A$. Set $\mathbb{C}_{0}(t) \equiv C^{-(n-1)} \mathbb{C}_{n}(t)$ for $t \in[-n \tau, n \tau]$ and $n \in N$ to yield

$$
A \int_{0}^{t}(t-s) \mathbb{C}_{0}(s) x d s=\mathbb{C}_{0}(t) x-C x, \forall t \in R
$$

by (3.1) and (3.2). Thus, $\left\{\mathbb{C}_{0}(t)\right\}_{t \in R}$ is the global extension of $\left\{\mathbb{C}_{1}(t)\right\}_{t \in[-\tau, \tau]}$. If the latter is for (resp. generated by) $A$, then the former is also by its definition and Theorem 3.6.

It is worthwhile to mention that for regularized cosine functions, we have results similar to Corollaries 2.9, 2.10 and Remark 2.11. Since all of these are easily verified, we omit the details.

\section{EXAMPLES}

In this last section we present several examples.

The following Example 4.1 was suggested by the referee.

Example 4.1. (a) Let $\left\{e^{-t A}\right\}_{t \geq 0}$ be a strongly continuous semigroup generated by $-A$. Let $C \equiv e^{-\tau A}$, where $\tau>0$ is given. Then

$$
W(t) \equiv e^{(t-\tau) A} \quad(0 \leq t \leq \tau)
$$

defines a local $C$-regularized semigroup generated by $A$. The local $C^{n}$-regularized semigroup $W_{n}(t) \equiv e^{(t-n \tau) A}$ is the extension of $W(\cdot)$ to $t \in[0, n \tau]$ for $n \in N$.

(b) Let $A, C$ and $\tau$ be as in (a). Then

$$
\mathbb{C}(t) \equiv \frac{1}{2}\left[e^{(t-\tau) A}+e^{-(t+\tau) A}\right], \quad t \in[-\tau, \tau]
$$

defines a local $C$-regularized cosine function generated by $A^{2}$. The local $C^{n_{-}}$ regularized cosine function

$$
\mathbb{C}_{n}(t) \equiv \frac{1}{2}\left[e^{(t-n \tau) A}+e^{-(t+n \tau) A}\right], \quad t \in[-n \tau, n \tau]
$$

is the extension of $\mathbb{C}(\cdot)$ to $t \in[-n \tau, n \tau]$ for $n \in N$.

(a) is interesting because it focuses on reversibility of solutions of abstract Cauchy problems of the first order. It implies that if the following abstract Cauchy problem

$$
\left\{\begin{array}{l}
\frac{d}{d t} u(t)+A u(t)=0, t \geq 0 ; \\
u(0)=x
\end{array}\right.
$$

has a unique exponentially bounded mild solution for every $x \in X$, then it has a unique mild solution on $[-n \tau, n \tau]$ for every $x \in \operatorname{Im}\left(e^{-n \tau A}\right)$ with $n \in N$.

Example 4.2. (a) Let $A$ be a (possibly unbounded) scalar type operator on the Banach space $X$ and let $K(\cdot)$ be the resolution of the identity for $A$. Then

$$
A x=\int_{\sigma(A)} \lambda K(d \lambda) x, \forall x \in D(A) .
$$

Given $\tau>0$. Assume there is $r_{0} \in R$ such that $\sigma(A) \subseteq\left\{\lambda \mid \lambda \in \mathcal{C}, \operatorname{Re}(\lambda) \geq r_{0}\right\}$. If $K((-i \infty, i \infty))=0$, then

$$
C \equiv \int_{\sigma(A)} \operatorname{Re}(\lambda) e^{-\tau \lambda} K(d \lambda)
$$


is bounded and injective, and

$$
W(t) \equiv \int_{\sigma(A)} \operatorname{Re}(\lambda) e^{\lambda(t-\tau)} K(d \lambda)
$$

defines the local $C$-regularized semigroup $\{W(t)\}_{t \in[0, \tau)}$ generated by $A$. If $\lambda \mapsto$ $\operatorname{Re}(\lambda)$ is not essentially bounded on $\sigma(A)$ with respect to the vector measure $K(\cdot)$, then $\{W(t)\}_{t \in[0, \tau)}$ cannot be extended to $t \geq \tau$. However, Remark 2.11 implies that

$$
W_{n}(t)=\int_{\sigma(A)}[R e(\lambda)]^{n} e^{\lambda(t-n \tau)} K(d \lambda)(t \in[0, n \tau)
$$

defines a local $C^{n}$-regularized semigroup $\left\{W_{n}(t)\right\}_{t \in[0, n \tau)}$ generated by $A$.

(b) Let $\tau, A$ and $K(\cdot)$ be as in (a). Then

$$
\mathbb{C}(t) \equiv \frac{1}{2} \int_{\sigma(A)} \operatorname{Re} \lambda\left[e^{\lambda(t-\tau)}+e^{-\lambda(t+\tau)}\right] K(d \lambda)(t \in(-\tau, \tau))
$$

defines a local $C$-regularized cosine function $\{\mathbb{C}(t)\}_{t \in(-\tau, \tau)}$ generated by $A^{2}$ and

$$
\mathbb{C}_{n}(t)=\frac{1}{2} \int_{\sigma(A)}[\operatorname{Re}(\lambda)]^{n}\left[e^{\lambda(t-n \tau)}+e^{-\lambda(t+n \tau)}\right] K(d \lambda)(t \in(-n \tau, n \tau))
$$

and defines a local $C^{n}$-regularized cosine function also generated by $A^{2}$.

Example 4.3. Let $A$ be a (possibly unbounded) scalar type operator on $X$ and let $K(\cdot)$ be the resolution of the identity for $A$. Assume $\rho(A)$ is nonempty.

(a) Let $k \in N$ and $r \in \rho(A)$. Then

$$
(A-r)^{-k}=\int_{\sigma(A)}(\lambda-r)^{-k} K(d \lambda)
$$

is bounded and injective. As with [A-El-K], for any given $\alpha>0, \beta>0$, denote

$$
E(\alpha, \beta) \equiv\left\{\lambda|\lambda \in \mathcal{C}, \operatorname{Re}(\lambda) \geq \beta,| \operatorname{Im}(\lambda) \mid \leq e^{\alpha \operatorname{Re}(\lambda)}\right\} .
$$

Assume $E(\alpha, \beta) \subseteq \rho(A)$ and let $r \in \rho(A)$ be real. For $\lambda \in \sigma(A)$ with $\operatorname{Re}(\lambda) \geq \beta$, we have

$$
|\lambda-r|^{k} \geq|\operatorname{Im}(\lambda)|^{k} \geq e^{\alpha k \operatorname{Re}(\lambda)} \geq e^{\tau \operatorname{Re}(\lambda)},
$$

where $\tau$ satisfies $0<\tau \leq \alpha k$. (4.1) implies that

$$
W(t) \equiv \int_{\sigma(A)}(\lambda-r)^{-k} e^{\lambda t} K(d \lambda)
$$

defines a local $(A-r)^{-k}$-regularized semigroup generated by $A$ and

$$
W_{n}(t)=\int_{\sigma(A)}(\lambda-r)^{-n k} e^{\lambda(t-n \tau)} K(d \lambda) \quad(t \in[0, n \tau))
$$

defines a $(A-r)^{-n k}$-regularized semigroup generated by $A$.

(b) Let $k, E(\alpha, \beta), r$ and $\tau$ be as in (a). From (4.1),

$$
\mathbb{C}(t) \equiv \frac{1}{2} \int_{\sigma(A)}(\lambda-r)^{-k}\left[e^{\lambda t}+e^{-\lambda t}\right] K(d \lambda) \quad(t \in[-\tau, \tau])
$$

defines a local $(A-r)^{-k}$-regularized cosine function generated by $A^{2}$ and

$$
\mathbb{C}_{n}(t)=\frac{1}{2} \int_{\sigma(A)}(\lambda-r)^{-n k}\left[e^{\lambda t}+e^{-\lambda t}\right] K(d \lambda) \quad(t \in[-n \tau, n \tau])
$$


defines a local $(A-r)^{-n k}$-regularized cosine function generated by $A^{2}$.

To present our last example, we need the following Definitions 4.4 and 4.5 which can be found in [dL 1] or [dL $4-\mathrm{Wa}]$.

Definition 4.4. Let $\Omega \subseteq \mathcal{C}$ be open so that the complement $\mathcal{C}-\Omega$ contains a half line, and let $\alpha \geq-1$. The operator $A$ is of $\alpha$-type $\Omega$ if $\sigma(A) \subseteq \Omega$ and there exists a constant $M>0$ so that

$$
\left\|(\lambda-A)^{-1}\right\| \leq M(1+|\lambda|)^{\alpha}, \forall \lambda \notin \Omega .
$$

For a given $m \in N \cup\{0\}$, let $H_{m}^{\infty}(\Omega)$ be the set of all functions $f: \Omega \mapsto \mathcal{C}$ so that

$$
z \mapsto(\lambda-z)^{m} f(z) \in H^{\infty}(\Omega), \forall \lambda \notin \bar{\Omega} .
$$

Definition 4.5. Assume $A$ is of $\alpha$-type $\Omega, m \equiv[\alpha]+2$ and $h \in H_{m}^{\infty}(\Omega)$. Define $h(A) \in B(X)$ by

$$
h(A) \equiv \frac{1}{2 \pi i} \int_{\partial O} h(w)(w-A)^{-1} d w,
$$

where $O$ is chosen so that $\bar{O} \subset \Omega$ and $A$ is of $\alpha$-type $O$ (see [dL 1, Lemma 22.4]).

Let $\tau>0$ and let $\mathbb{E}_{\Omega, \tau}$ be the set of all functions $h: \Omega \mapsto \mathcal{C}$ so that

$$
z \mapsto e^{t z} h(z) \in H^{\infty}(\Omega)
$$

for all $t \in[0, \tau]$.

Example 4.6. Assume $A$ is of $\alpha$-type $\Omega, m \equiv[\alpha]+2$.

(a) If there exists $g \in \mathbb{E}_{\Omega, \tau} \cap H_{m}^{\infty}(\Omega)$, then

$$
W(t) \equiv\left(z \mapsto e^{t z} g(z)\right)(A) \quad(t \in[0, \tau])
$$

defines a local $g(A)$-regularized semigroup for $A$.

(b) $g(A)$ may be chosen nontrivial, if we further assume that

(i) $\mathbb{E}_{\Omega, \tau}$ is nontrivial;

(ii) $\sigma(A) \cap \Omega_{0} \neq \emptyset$ for every connected component $\Omega_{0}$ of $\Omega$.

(c) $g(A)$ may be chosen injective provided

(iii) $A$ is a quasi-generalized scalar operator (see [Wa 2, Definition 2.5]) and

(iv) there exists $k \in \mathbb{E}_{\Omega, \tau}$ such that $k(\mu) \neq 0$ for all $\mu \in \sigma(A)$.

Proof. The proof of (a) and (b) is almost identical to that of [dL 4-Wa, Theorem 2.3]. Now we prove (c). Let $k \in \mathbb{E}_{\Omega, \tau}$ so that $k(\mu) \neq 0$ for all $\mu \in \sigma(A)$. Define

$$
g(z) \equiv \frac{k(z)}{(\lambda-z)^{m}} .
$$

Then $g \in \mathbb{E}_{\Omega, \tau} \cap H_{m}^{\infty}(\Omega)$. If $g(A)$ is not injective, then $\mathbb{N} \equiv k \operatorname{ker}(g(A))$ is nontrivial. From $g(A) A \subseteq A g(A)$, we have

$$
g(A)(\lambda-A)^{-1}=(\lambda-A)^{-1} g(A)
$$

for all $\lambda \in \rho(A)$. This implies that $g(A)$ is commutative with $\varepsilon$ by (4.2), since $\varepsilon$ is commutative with $(\lambda-A)^{-1}$ for all $\lambda \in \rho(A)$, where $\varepsilon$ is a quasi-spectral distribution for $A$ (see [Wa 2, Definition 2.5]). Hence, $\mathbb{N}$ is invariant under $\varepsilon$ and $A \mid \mathbb{N}$ is a quasi-generalized scalar operator. Consequently, $\sigma(A \mid \mathcal{N})$ is nonempty (see [Wa 2]). $g(A)$ being the zero operator on $\mathbb{N}$, [dL 4-Wa, Proposition 3.2] implies that $k(\mu)=g(\mu)(\lambda-\mu)^{-m} \equiv 0$ for all $\mu \in \sigma(A \mid \mathbb{N})$. Since the latter is a nonempty subset of $\sigma(A)$ by (4.3), we are led to a contradiction. $g(A)$ is thus injective. 
Some other sufficient conditions guaranteeing $g(A)$ to be injective can be found in [dL 5, Theorem 5.2].

Open question. Do there exist functional calculi to characterize local regularized semigroups and regularized cosine functions (proposed by Ralph deLaubenfels in his letter)?

\section{ACKNOWLEDGEMENT}

The authors would like to highly thank the referee for many important suggestions and offering reference [dL 5].

\section{REFERENCES}

[A-El-K] W. Arendt, O. El-Mennaoui and V. Keyantuo, Local Integrated Semigroups: Evolution with jumps of Regularity, J. Math. Anal. Appl. 186 (1994,), 572-595. MR 95f: 47065

[dL 1] R. deLaubenfels, Existence Families, Functional Calculi and Evolution Equations, Lecture Notes in Math., Springer-Verlag, 1570, 1994. MR 96b:47047

[dL 2] R. deLaubenfels, Existence and Uniqueness Families for the abstract Cauchy problem, J. London Math. Soc. 44 (1991), 310-338. MR 92k:34075

[dL 3-S-Wa] R. deLaubenfels, G. Sun and S. W. Wang, Regularized Semigroups, Existence Families and the abstract Cauchy problem, Diff. Int. Eq. 8 (1995), 1477-1496. MR 96j: 47035

[dL 4-Wa] R. deLaubenfels, S. W. Wang, Spectral Conditions guaranteeing a Non-trivial Solution of the abstract Cauchy problem, Proc. Amer. Math. Soc., to appear.

[dL 5] R. deLaubenfels, Automatic extensions of functional calculi, Studia Math. 114 (1995), 237-259. MR 96f:47029

[F] H. O. Fattorini, Existence and behavior at infinity of solutions of certain linear operational differential equations, Pacific J. Math. 33 (1970), 583-615. MR 41:8789

[G] M. C. Gao, Local C-semigroups and Local C-cosine functions, preprint.

[H-Hu] F. Huang, T. Huang, Local C-cosine family theory and application, Chinese Ann. Math. Ser. 16B (1995), 213-232. MR 96g:47040

[L] J. L. Lions, Les Semi-groupes Distributions, Portugal. Math. 19 (1960), 141-164. MR 26:611

[S] G. Sun, Integrated C-semigroups, Local C-semigroups, Mild C-existence families and $(A C P)$, Dissertation (Chinese).

[T-O] N. Tanaka, N. Okazawa, Local C-Semigroups and Local Integrated Semigroups, Proc. London Math. Soc. 61 (1990), 63-90. MR 91b:47093

[W-Wa] H. Wang, S. W. Wang, C-Cosine Operator Functions and the Applications to the Second Order Abstract Cauchy Problem, Functional Analysis in China, Kluwer Acad. Publ. (Netherlands) 1996.

[Wa 1] S. W. Wang, Quasi-distribution Semigroups and Integrated Semigroups, J. Funct. Anal. 146 (1997), 352-381. MR 98d:47088

[Wa 2] S. W. Wang, Strongly Continuous Groups and Semigroups of Generalized Scalar Operators, submitted.

Department of Mathematics, Nanjing University, Nanjing, Jiangsu 210093, People's Republic of China

E-mail address: wang2598@netra.nju.edu.cn

Current address, Sheng Wang Wang: Department of Mathematics, Central Michigan University, Mt. Pleasant, Michigan 48859

Current address, Min Chu Gao: Department of Mathematics, Shanxi Teachers University, Linfen, Shanxi 041000, People's Republic of China 